\title{
Demographic and Clinical Features of Patients with Dengue in Northeastern Region of India: A Retrospective Cross-Sectional Study during 2009-2011
}

\author{
Prafulla Dutta, Siraj A. Khan, Jani Borah and Jagadish Mahanta \\ Regional Medical Research centre, North-east Region, ICMR, Assam, India
}

\begin{abstract}
The incidence of fever cases of unknown origin has increased in Northeastern Region of India in recent years. We conducted passive surveillance and performed laboratory testing on suspected cases for the presence of dengue infection during 2009-2011. We estimated the incidence by applying age, sex and season adjusted dengue positivity. We identified 430 suspected cases of which 143 (33.3\%) were found to be laboratory-confirmed dengue cases. Dengue transmission was found to occur in post-monsoon season (September-December). We evaluated the differences of clinical features and hematologic abnormalities that occurred in laboratory dengue positive and negative cases. Significantly higher percentage of dengue patients had headache, eye pain and vomiting $(P<0.05)$. Diarrhoea as well as abdominal pain was observed more commonly in laboratory dengue negative patients but the difference was not significant $(P>0.05)$. Thrombocytopenia was observed in significantly higher percentage of positive patients $(P<0.001)$ with significantly lower platelets counts $(P<0.001)$. The elevated level of Aspartate transaminase $(P<0.001)$ and Alanine transaminase $(P=0.002)$ was also significantly higher side in positive patients. This study provides some important information in clinical and laboratory data that can be used for early diagnosis and subsequently for appropriate treatment of dengue infections. Thus, the passive surveillance and laboratory confirmation provides a better estimate of the actual dengue disease burden and a scope to further refine dengue prevention strategies.
\end{abstract}

Key words: Dengue, Northeast India, Clinical features, Passive surveillance.

\section{Introduction}

Emerging viral infections have become a serious problem in recent years. Emergence or reemergence of severe arboviral hemorrhagic fevers caused by mosquito borne viruses, such as dengue virus (DENV) has been frequently reported in the Indian subcontinent in the past few years. Epidemic dengue fever was common in Asia and Pacific including India throughout the twentieth century (Gubler, 1998). The demographic features of dengue have changed tremendously in South East Asia over the past two decades (Khan et al., 2010). However, there is scarcity of data looking at the overall trend of dengue infection in the country. Infection with DENV can cause a spectrum of illness ranging from no symptoms to life-threatening dengue hemorrhagic fever/dengue shock syndrome. Dengue infection produces a self-limiting illness in humans that is often characterized by sudden onset of fever, headache, fatigue, nausea, vomiting, rash and myalgia (Bharaj et al., 2008; Silva et al., 2012).

Copyright (C) 2012Prafulla Dutta, Siraj A. Khan, Jani Borah and Jagadish Mahanta. This is an open access article distributed under the Creative Commons Attribution License unported 3.0, which permits unrestricted use, distribution, and reproduction in any medium, provided that original work is properly cited. Contact author: Prafulla Dutta E-mail: duttaprafulla@yahoo.com 
The causative agent of dengue is DENV (DENV-1, DENV-2, DENV-3 and DENV-4), a single-stranded, positive sense RNA, enveloped virus, is a member of the genus Flavivirus of the Flaviviridae family. It is generally transmitted to humans via Aedes aegypti and Aedes albopictus mosquitoes [Dutta et al., 2006; Kamgang et al., 2011].

The north-eastern region of India comprises of eight states, viz. Assam, Arunachal Pradesh, Manipur, Mizoram, Meghalaya, Nagaland, Tripura and Sikkim. DENV activity has been documented in Assam (Rodrigues and Dandawate, 1977; Dutta et al., 2011), Arunachal Pradesh (Rodrigues and Dandawate, 1977) and Nagaland (Dutta et al., 2006). Entomological survey carried out in different time periods reveals the prevalence of potential dengue vectors in this region (Khan et al., 1996; Dutta et al., 1998; Dutta et al., 1999; Dutta et al., 2004; Dutta et al., 2010). A comprehensive entomological survey conducted during 2004-2005 in the seven states of the northeastern (NE) region of India revealed that the region is rich in known dengue vectors, viz. Aedes (Stegomyia) aegypti and Aedes (Stegomyia) albopictus (Dutta et al., 2006). The different breeding habitats with preponderance of Aedes vector was recorded in four different environmental settings (Urban, Industrial, Semi-urban and rural) in all the states of Northeastern region (Dutta P et al., 2006).

The states of Northeast have experienced an increased number of reported fever cases of unknown origin in recent years. The doctors rarely consider dengue as a differential diagnosis of an acute febrile illness. The signs and symptoms of dengue being non-specific, the physician must maintain a high index of suspicion if a clinical diagnosis of dengue is to be made (Gubler, 1998; Kuo et al., 2007). The clinical manifestation of dengue and a complete medical history for early diagnosis are important for prompt supportive therapy. Dengue shared some similar symptoms with other infectious diseases prevalent in this region viz. Chikungunya (Dutta et al., 2011), West Nile (Khan et al.,
2011) and Japanese encephalitis (Borah et al., 2011). Thus, most of the dengue infections go undiagnosed and under reported.

In the current study, we have looked on the dengue cases for better understanding of its incidence and epidemiology in Northeast region. The primary objective of this study was to determine the demographic characteristics, clinical features, and estimate the incidence of dengue in the Northeast region during 2009-2011. This is a maiden study on dengue infection in this part of the country which is very important to design prevention and control strategy accordingly.

\section{Methods}

\section{Clinical Samples}

The study included 430 patients with a history of sudden onset of fever and the presence of two or more of the symptoms viz. headache, eye pain, nausea, vomiting, rash, myalgia, abdominal pain. Acute-phase serum samples were obtained from the patients either admitted to the Government or private hospitals of Assam and from those who were referred by treating clinician to the Regional Medical Research Centre, Assam, India for diagnosis from different Northeastern states of India during the period from January'2009 to January'2011. There was no sampling bias or any attempt to specifically recruit patients. The exclusion criteria includes: if routine lab tests, i.e. platelet count, complete blood count, malaria parasite, chest X-ray, etc., were already done and those reports suggested bacterial infections or any clinical diagnosis other than viral infections and if the patient or his/her guardian refused to participate in the survey. Serum specimens were aliquoted in sterile vials and stored at $80^{\circ} \mathrm{C}$ until use. Written informed consent was obtained from each patient. The clinical symptoms including the laboratory findings were recorded on case record forms. The study was approved by the Institutional Ethics Committee of Regional Medical 
Research Centre, ICMR, Northeast region, Dibrugarh, Assam, India.

\section{Serological Testing}

Serum specimens obtained from these 430 patients were tested for the presence of DENV-specific IgM antibodies using MAC ELISA kit obtained from National Institute of Virology (NIV), ICMR, Pune. The samples that exhibited OD value more than 5 times than that of negative control were considered as positive for acute dengue infection. The DENV-IgM positive samples were further tested for the presence of IgM antibody against other flaviviruses viz. Japanese encephalitis (MAC ELISA kit, NIV, ICMR, Pune) and West Nile (MAC ELISA kit, PanBio, Australia) prevalent in this region. All the serum samples were also subjected for detection of IgG antibodies by using IgG capture ELISA kit (PanBio, Australia) and NS1 antigen by using Platelia Dengue NS1 Ag kits (Bio-Rad, USA).

\section{Molecular Assays}

The IgM negative serum samples were tested for the presence of DENV-specific RNA by RT-PCR (Harris et al., 1998). RNA was extracted using the QIAamp Viral RNA Mini Kit (Qiagen). Two step RT-PCR was performed. cDNA was synthesized by using cDNA synthesis kit (Fermentas). PCR was done by employing primer pairs targeting the Capsid gene (Lanciotti et al., 1992) using $2 \mu \mathrm{l}$ cDNA as template.

\section{Data Analysis}

Statistical Package for Social Sciences (SPSS) version 15.0 (Chicago, IL) was used for data entry, processing and statistical analysis at the end of the study period. Descriptive statistics were used to calculate all the relevant variables. Differences of demographic and clinical characteristics between laboratory confirmed dengue cases and negative cases were evaluated using the $\chi 2$ test with Yates' correction (Epi-Info version 6.6). Differences in means were assessed by Student's t-test. A $P$ value of less than 0.05 was considered as significant.

\section{Results}

Of the 430 subjects screened, 143 (33.3\%) were confirmed as having DENV infection: 92 $(64.3 \%)$ by IgM-capture ELISA and 51 $(35.7 \%)$ by RT-PCR. The DENV-IgM positive samples were found to be negative for the presence of IgM against other flaviviruses viz. Japanese encephalitis and West Nile prevalent in this region. All the RT-PCR positive samples were found to be positive for DENV NS1 antigen. Out of 51 RT-PCR positive samples, 28 were found to be positive for IgG antibodies also. Hence, of the 430 subjects screened against dengue, primary dengue infection was observed in 115 acute phase sera samples and secondary infection was observed in 28 sera samples. Out of the 143 laboratory-confirmed cases, 23 patients were enrolled in 2009, 94 in 2010, and 26 in 2011. Among the dengue cases, there were 82 from Assam, 35 from Meghalaya, 15 from Nagaland, 8 from Manipur and 3 from Arunachal Pradesh. The seasonality of dengue cases pertaining to the highest proportion of seropositivity was observed during post monsoon season (September-December) in the year 2009 and 2010. However, in 2011, the highest proportion of cases was observed in April (Figure 1). 

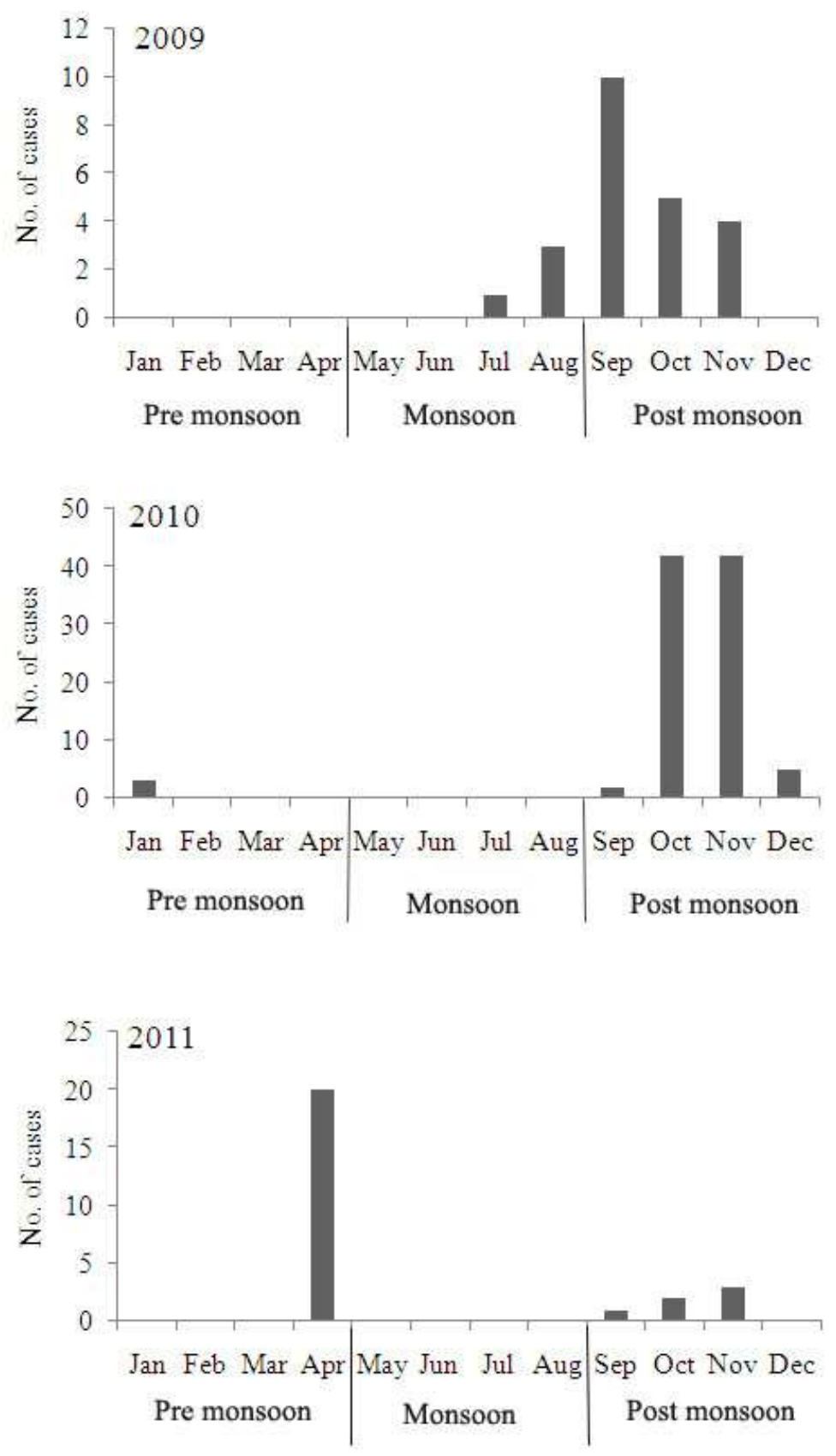

Figure 1: Distribution of Dengue Cases by Season and Year of Enrollment. The Number of Laboratory Confirmed Dengue Cases is plotted at Monthly Intervals from January 2009 to December 2011 
The distribution of all the four DENV serotypes (DENV-1, DENV-2, DENV-3 and DENV-4) was found in Northeast region. DENV-1 was the dominant serotypes in this study population and accounted for $45.1 \%$ $(23 / 51)$ of viraemic patients. Concurrent infections with DENV-1 and 2 (3 nos.), DENV-
1 and 3 (1 no.), DENV-1 and 4 (1 no.) and DENV-2 and 3 (3 nos.) were observed.

The socioeconomic and demographic profiles of the laboratory positive dengue and laboratory negative dengue cases have been given in Table 1.

Table 1: Demographic Characteristics of Laboratory Dengue Positive and Negative Patients

\begin{tabular}{|c|l|l|l|l|}
\hline \multirow{2}{*}{ Characteristices } & \multicolumn{2}{|l|}{ No. (\%) } & \multirow{2}{*}{$P$} \\
\cline { 2 - 4 } & $\begin{array}{l}\text { Laboratory dengue } \\
\text { positive cases }\end{array}$ & \multicolumn{1}{|l|}{$\begin{array}{l}\text { Laboratory dengue negative } \\
\text { cases }\end{array}$} & \\
\cline { 2 - 4 }$(\mathrm{n}=143)$ & & & $(\mathrm{n}=287)$ \\
\hline Age (years) & & & 0.213 \\
\hline Male & $96(67.1)$ & & $212(73.9)$ & \\
\hline Gender & $47(32.9)$ & & & 0.079 \\
\hline Female & $82(57.3)$ & & $205(71.4)$ & \\
\hline Place of residence & $61(42.7)$ & & $82(28.6)$ & 0.126 \\
\hline Rural & $93(65)$ & & $217(75.6)$ & \\
\hline Urban & $50(35)$ & & $70(24.4)$ & 0.481 \\
\hline Literacy status & & & \\
\hline Illiterate & $88(61.5)$ & & $197(68.6)$ & \\
\hline Literatte & $55(38.5)$ & & $90(31.4)$ & \\
\hline
\end{tabular}

Substantial proportions of dengue cases were found to occur among male (57.3\%) and children $\leq 15$ years of age $(67.1 \%)$. When the percentage of laboratory dengue positive cases was compared with that of laboratory negative cases, no significant differences were observed in case of age, sex, place of residence, and literacy. Illiterate male patients were found predominant in both the groups. Most of the patients with dengue and other febrile illness belonged to rural background.

The clinical features exhibited by the laboratory positive and negative dengue cases have been shown in Table 2 . 
Table 2: Clinical Characteristics of Laboratory Dengue Positive and Negative Patients Hospitalized During 2009-2011

\begin{tabular}{|l|l|l|l|}
\hline \multirow{2}{*}{ Characteristices } & \multicolumn{2}{|l|}{ No. $(\%)$} & \multirow{2}{*}{ dengue } \\
\cline { 2 - 3 } & $\begin{array}{l}\text { Laboratory dengue positive } \\
\text { cases }\end{array}$ & $\begin{array}{l}\text { Laboratory } \\
\text { negative cases }\end{array}$ & \\
\cline { 2 - 3 }$(\mathrm{n}=143)$ & (n=287) & 0.924 \\
\hline Fever & $143(100)$ & $285(99.3)$ & 0.001 \\
\hline Headache & $124(86.7)$ & $97(33.8)$ & 0.146 \\
\hline Myalgia & $87(60.8)$ & $143(49.8)$ & 0.004 \\
\hline Eye pain & $98(68.5)$ & $134(46.6)$ & 0.454 \\
\hline Diarrhoea & $65(45.5)$ & $153(53.3)$ & 0.013 \\
\hline Nausea & $54(37.7)$ & $106(33.8)$ & 0.106 \\
\hline Vomiting & $78(54.5)$ & $168(58.5)$ & - \\
\hline Abdominal pain & $76(53.1)$ & - & \\
\hline Rash & $8(5.6)$ & & \\
\hline
\end{tabular}

Both laboratory-positive and laboratorynegative patients sought medical care a median of 5 days after the onset of symptoms. Clinical symptoms of laboratoryconfirmed dengue cases were consistent with classical descriptions as high proportions of fever, headache, eye pain and vomiting. Fever was the most common clinical feature in almost all the positive (100\%) and negative patients $(99.3 \%)$. The other most common presenting features of laboratory dengue positive patients were headache $(86.7 \%)$, eye pain $(68.5 \%)$ and myalgia $(60.8 \%)$. However, abdominal pain $(58.5 \%)$ and diarrhoea (53.3\%) were most commonly observed symptoms in laboratory dengue negative patients. The proportion of cases with headache $(86.7 \%$ vs $33.8 \%, P<0.001)$, eye pain (68.5 \% vs $46.6 \%, P=0.004)$ and vomiting (54.5\% vs $36.9 \%, P=0.013$ ) was significantly higher in laboratory dengue positive cases in comparison to negative patients. Hemorrhage was observed in 8 numbers of laboratory-positive cases. None of the laboratory-negative cases showed the symptoms of hemorrhages.

A comparison of laboratory findings during hospitalization is presented in Table 3 .

Table 3: Summary of Findings of Laboratory Dengue Positive and Negative Cases

\begin{tabular}{|c|c|c|c|c|c|c|}
\hline & \multicolumn{3}{|l|}{ No. (\%) } & \multicolumn{3}{|l|}{ Mean \pm SD } \\
\hline \multirow[t]{2}{*}{ Characteristics } & $\begin{array}{l}\text { Laboratory } \\
\text { dengue } \\
\text { positive } \\
\text { cases }\end{array}$ & $\begin{array}{l}\text { Laboratory } \\
\text { dengue } \\
\text { negative } \\
\text { cases }\end{array}$ & $P$ & $\begin{array}{l}\text { Laboratory } \\
\text { dengue } \\
\text { positive } \\
\text { cases }\end{array}$ & $\begin{array}{l}\text { Laboratory } \\
\text { dengue } \\
\text { negative } \\
\text { cases }\end{array}$ & $P$ \\
\hline & $(n=143)$ & $(n=287)$ & & $(n=143)$ & $(n=287)$ & \\
\hline $\begin{array}{l}\text { Thrombocytopenia, } \\
<1.5 \times 105 / \mathrm{mm} 3\end{array}$ & $102(71.3)$ & $5(1.7)$ & $<0.001$ & $\begin{array}{l}55342 \quad \pm \\
45300\end{array}$ & $\begin{array}{l}143342 \quad \pm \\
32454\end{array}$ & $<0.001$ \\
\hline $\begin{array}{l}\text { Leucopenia, }<4,000 \\
\text { cells/mm3 }\end{array}$ & $43(30)$ & $78(27.2)$ & 0.623 & $3254 \pm 1323$ & $3645 \pm 1654$ & 0.325 \\
\hline $\begin{array}{l}\text { Aspartate } \\
\text { transaminase, }>40 \\
\mathrm{U} / \mathrm{L}\end{array}$ & $98(68.5)$ & $64(22.3)$ & 0.002 & $75.3 \pm 44.6$ & $45.2 \pm 21.4$ & $<0.001$ \\
\hline $\begin{array}{l}\text { Alanine } \\
\text { transaminase, }>40 \\
\mathrm{U} / \mathrm{L}\end{array}$ & $56(39.2)$ & $34(11.8)$ & 0.026 & $63.6 \pm 34.5$ & $58.5 \pm 28.9$ & 0.766 \\
\hline
\end{tabular}


A significantly higher percentage of laboratory positive patients $(71.3 \%$ vs. $1.7 \%$, $P<0.001$ ) showed lower platelet counts $(55342 \pm 45300$ vs. $143342 \pm 32454, P<$ 0.001) when compared with laboratory negative dengue cases. The percentage value of laboratory positive cases with elevated Aspartate transaminase $(68.5 \%$ vs. $22.3 \%, P$ $=0.0020 .01$ ) and Alanine transaminase (39.2\% vs. $11.8 \%, \mathrm{P}<0.05)$ was significantly higher. The mean elevated level of Aspartate transaminase was significantly higher $(P<$ 0.001 ) in laboratory dengue positive patients but the difference in the elevated level of Alanine transaminase between both the groups was not significant $(P=0.766)$. No significant difference in leukocyte count at presentation was seen.

\section{Discussion}

DENV presently threatens half of the world's population and is an important public health problem in many tropical regions of the world (Strobel and Lamaury, 2001). In the last three decades, the demographic and clinical features of dengue infections have changed rapidly (khan et al., 2010). To know the actual picture of the disease in a large scale, it is important to study the incidence of a particular disease in a region wise manner. This is the first effort in Northeast region to perform surveillance for dengue supported by comprehensive laboratory testing for further prevention of the spread of the diseases. During the three years from 2009 to 2011, we identified 430 suspected cases. Among them, $33.3 \%$ were laboratory confirmed dengue cases. The incidence of laboratory-confirmed dengue was predominant in young children. In the present study, $67.1 \%$ cases were $\leq 15$ years of age. This finding probably suggests that there is indeed a lack of herd immunity to DENV. This lack of herd immunity in younger population probably accounts for affecting the younger ones $(\leq 15 \mathrm{yr})$ than elderly $(>15$ yr) ones (32.9\%) in our study. Sex distribution shows a male preponderance in our study and this finding is in concordance with that of an earlier study (Tripathi et al., 2007).

Dengue has traditionally been held to be a disease of high population density tropical urban areas (Pavri, 1977; Teixeira et al., 2002). However, increasing reports of dengue cases and outbreaks from rural areas were reported from northern, southern and western India (Kumar et al., 2001; Norman et al., 1991; Ilkal et al., 1991). The findings pertaining to the first kind of study from the Northeastern region provide similar picture with other parts of the country. The cut bamboo stumps, tree holes, plant leaf axils, discarded coconut shells and glass bottles are the natural habitats that store rain water facilitating breeding of potential dengue vectors in and around the rural dwellings. Simple source reduction methods through community efforts and awareness campaign may help in effective elimination of the vector population and further control of disease (Dutta et al., 2006).

The epidemics of dengue have been commonly associated with the rainy season (Keating, 2001). In a study conducted in Lucknow, India and Pakistan, it was observed that dengue transmission occurred round the year with peak incidence in the postmonsoon season (Tripathi et al 2008; Khan et al., 2010). Similarly, these studies found the highest proportion of dengue positive patients during post monsoon season. But in the present study most of the cases were found to report during pre monsoon season during 2011. Factors responsible for such shifting need to be identified and addressed in future studies.

No significant difference in occurrence of dengue was identified in age, sex, place of residence and literacy status among the positive and negative patients suggesting that the demographic characteristics in both the group are identical. In the present context, majority of patients of both the age groups were illiterate. 
The illiterate people are not aware about the transmission of dengue. Information, Education and Communication (IEC) activities/awareness campaigns are needed to motivate the local illiterate populace of risk areas to take protective measures against further transmission of this disease (Dutta et al., 2011).

The findings herein serve to demonstrate the differences in clinical and laboratory features in dengue positive and negative cases during admission. All the patients suffered from fever irrespective of their dengue positivity. Since fever was found in all the suspected cases, a diagnostic value of this symptom for this disease is limited. The percentage of positive cases having headache, eye pain and vomiting were significantly higher during the acute phase of the illness than that in the negative cases. These common clinical features in typical dengue infection were also observed in other published studies (PérezGuerra et al., 2005; Chuang et al., 2008; Lee et al., 2012). Our laboratory data showed that the percentage of dengue patients with mean elevated level of Aspartate transaminase and Alanine transaminase were significantly higher than that of laboratory dengue negative patients. Previous studies suggested that liver injury is a common finding in dengue infections and it is mediated by direct infection of hepatocytes and Kupffer cells (Murgue et al., 1999; Souza et al., 2007). Recently, Luiz et al suggested the use of markers such as Aspartate transaminase and Alanine transaminase as parameters to evaluate severity in patients with dengue fever (Luiz et al., 2004). Since grossly elevated liver enzymes are known to be an early warning sign for severe disease and clinical bleeding, vigorous follow up in such patients is warranted (Murgue et al., 1999).

This study has provided important insights into the incidence and epidemiology of dengue in Northeast region. From this study, it is apparent that dengue surveillance and control should be enhanced by wider use of laboratory testing to confirm dengue as a cause of fever of unknown origin, especially during the local dengue transmission season. Control at source is one of the key for combating dengue fever and requires active participation from all sectors of the community. Our data also provide some important differences in the frequency of clinical symptoms between patients with dengue and undifferentiated febrile illness which in turn helps the clinicians to make suspicion for possible dengue viral infections in the differential diagnosis of febrile patients. Data from enhanced dengue surveillance should be used to further refine dengue prevention strategies. The present study also indicates that fever cases of unknown origin impose a considerable burden in the health care system. In recent years, increase of such cases in NE region of India is posing a challenge with regards to proper diagnosis, treatment and management of such cases as other diseases showing somewhat similar clinical picture with dengue viz. Japanese encephalitis (Borah et al., 2011), West Nile (Khan et al., 2011), Leptospirosis (Khan et al., 2012) and Scrub typhus (Khan et al., 2012) are also reported to be prevalent in this region. The other etiologies responsible for such burden need to be identified and addressed in future studies.

\section{Acknowledgements}

The authors are grateful to Chandra Kanta Sarmah, Pobitra Doloi, Niranjan Barua and Robin Doloi for their help and support during the study. Technical help received from Pritom Chowdhury, Rashmee Topno, Monika Soni, Lakshyajit Borah, Joy Gogoi are acknowledged with thanks.

\section{References}

Bharaj, P., Chahar, H. S., Pandey, A., Diddi, K., Dar, L., Guleria, R., Kabra, S. K. \& Broor, S. (2008). "Concurrent Infections by All Four Dengue Virus Serotypes During an Outbreak of Dengue in 2006 in Delhi, India," Virology Journal, 5(1). 
Borah, J., Dutta, P., Khan, S. A. \& Mahanta, J. (2011). "A Comparison of Clinical Features of Japanese Encephalitis Virus Infection in the Adult and Pediatric Age Group with Acute Encephalitis Syndrome," Journal of Clinical Virology, 52, 45- 49.

de Souza, L. J., Alves, J. G., Nogueira, R. M. R., Neto, C. G., Bastos, D. A., da Silva Siqueira, E. W., Filho, J. T. D. S., de Abreu Cezario, T., Soares, C. E. \& da Costa Carneiro, R. (2004). "Aminotransferase Changes and Acute Hepatitis in Patients with Dengue Fever: Analysis of 1,585 Cases," The Brazilian Journal of Infectious Diseases, 8(2), 156-163.

de Souza, L. J., Nogueira, R. M. R., Soares, L. C., Soares, C. E. C., Ribas, B. F. Alves, F. P., Vieira, F. R. \& Pessanha F. E. B. (2007). "The Impact of Dengue on Liver Function as Evaluated by Aminotransferase Levels," Brazilian Journal of Infectious Diseases, 11, 407-410.

Dutta, P., Khan, S. A., Khan, A. M., Borah, J., Chowdhury, P. \& Mahanta, J. (2011). "First Evidence of Chikungunya Virus Infection in Assam, Northeast, India," Transactions of the Royal Society of Tropical Medicine and Hygiene, 105, 355-357.

Dutta, P., Khan, S. A., Khan, A. M., Sharma, C. K., Doloi, P. K. \& Mahanta, J. (1999). "Solid Waste Pollution and Breeding Potential of Dengue Vectors in an Urban and Industrial Environment of Assam," Journal of Environmental Biology, 20 (4), 343-345.

Dutta, P., Khan, S. A., Khan, A. M., Sharma, C. K. \& Mahanta, J. (2004). "Entomological Observations on Dengue Vector Mosquitoes Following a Suspected Outbreak of Dengue in Certain Parts of Nagaland with a Note on Their Susceptibility to Insecticides," Journal of Environmental Biology, 25(2), 209-212.

Dutta, P., Khan S. A., Khan, A. M., Sharma, C. K. \& Mahanta, J. (2010). "Survey of Mosquito Species in Nagaland, A Hilly State of North East Region of India," Journal of Environmental Biology, 31 (5), 781-785.
Dutta, P., Khan, S. A., Sharma, C. K., Doloi, P. K., Hazarika, N. C. \& Mahanta, J. (1998). "Distribution of Potential Dengue Vectors in Major Townships Along the National Highways and Trunk Roads of Northeast India," Southeast Asian Journal of Tropical Medicine and Public Health, 29 (1), 173-176.

Dutta, P. \& Mahanta, J. (2006). 'Potential Vectors of Dengue and the Profile of Dengue in the Northeasrtern Region of India: An Epidemiological Perspective,' WHO Dengue Bulletin, 30, 234-242.

Gubler, D. J. (1998). "Dengue and Dengue Hemorrhagic Fever," Clinical Microbiology Review, 11, 480-496.

Harris, E., Roberts, T. G., Smith, L., Selle, J., Kramer, L. D., Valle, S., Sandoval, E. \& Balmaseda, A. (1998). "Typing of Dengue Viruses in Clinical Specimen and Mosquito by Single- Tube Multiplex Reverse Transcriptase-PCR," Journal of Clinical Microbiology, 36(9), 2634-2639.

Ilkal, M. A., Dhanda, V., Hassan, M. M., Mavale, M., Mahadev, P. V., Shetty, P. S., Guttikar, S. N. \& Banerjee, K. (1991). "Entomological Investigations During Outbreaks of Dengue Fever in Certain Villages in Maharashtra State," Indian Journal Medical Research, 93, 174-178.

Innis, B. L, Nisalak, A., Nimmannitya, S., Kusalerdchariya, S., Chongswasdi, V., Suntayakorn, S., Puttisri, P., Hoke, C. H. (1989). "An Enzyme-Linked Immunosorbent Assay to Characterize Dengue Infections Where Dengue and Japanese Encephalitis CoCirculate," American Journal of Tropical Medicine and Hygiene, 40(4), 418-27.

Kamgang, B., Marcombe, S., Chandre, F., Nchoutpouen, E., Nwane, P., Etang, J., Corbel, V. \& Paupy, C. (2011). "Insecticide Susceptibility of Aedes Aegypti and Aedes Albopictus in Central Africa," Parasites \& Vectors, 4, 79-86. 
Keating, J. (2001). "An Investigation into the Cyclical Incidence of Dengue Fever," Social Science and Medicine, 53, 1587-1597.

Khan, E., Kisat, M., Khan, N., Nasir, A., Ayub, S. \& Hasan, R. (2010). "Demographic and Clinical Features of Dengue Fever in Pakistan from 2003-2007: A Retrospective CrossSectional Study," Plos ONE, 5(9), E12505.

Khan, S. A., Dutta, P., Borah, J., Chowdhury, P., Topno, R., Baishya, M. \& Mahanta, J. (2012). "Leptospirosis Presenting as Acute Encephalitis Syndrome (AES) in Assam, India," Asian Pacific Journal of Tropical Disease, 151-153.

Khan, S. A., Dutta, P., Khan, A. M., Chowdhury, P., Borah, J., Doloi, P. \& Mahanta, J. (2011). "West Nile Virus Infection, Assam,India," Emerging Infectious Diseases, 17 (5), 947.

Khan, S. A., Dutta, P., Khan, A. M., Topno, R., Borah, J., Chowdhury, P. \& Mahanta, J. (2012). "Re-Emergence of Scrub Typhus in Northeast India," International Journal of Infectious Diseases. 16(12), 889-890.

Khan, S. A., Narain, K., Handique, R., Dutta, P., Mahanta, J., Satyanarayana, K. \& Srivastava, V. K. (1996). "Role of Some Environmental Factors in Modulating Seasonal Abundance of Potential Japanese Encephalitis Vectors in Assam, India," Southeast Asian Journal of Tropical Medicine and Public Health, 27 (2), 382-391.

Kumar, A., Sharma, S. K., Padbidri, V. S., Thakare, J. P., Jain, D. C. \& Datta, K. K. (2001). "An Outbreak of Dengue Fever in Rural Areas of Northern India," Journal of Communicable Disease, 33, 274-281.

Kuo, M. C., Chang, J. M., Po-Liang, L., Chiu, Y. W., Chen, H. C. \& Hwang, S. J. (2007). "Difficulty in Diagnosis and Treatment of Dengue Hemorrhagic Fever in Patients with Chronic Renal Failure: Report of Three Cases of Mortality," American Journal of Tropical Medicine and Hygiene, 76(4), 752-756.
Lanciotti, R. S., Calisher, C. H., Gubler, D. J., Chang, G. J. \& Vorndam, A. V. (1992). "Rapid Detection and Typing of Dengue Viruses from Clinical Samples by Using Reverse Transcriptase-Polymerase Chain Reaction," Journal of Clinical Microbiology, 30, 545-51.

Lee, L. K., Gan, V. C., Lee, V. J., Tan, A. S., Leo, Y. S. \& Lye, D. C. (2012). "Clinical Relevance and Discriminatory Value of Elevated Liver Aminotransferase Levels for Dengue Severity," Plos Neglated Tropical Disisease, 6(6), E1676.

Matheus, S., Deparis, X., Labeau, B., Lelarge, J., Movran, J. \& Dussart, P. (2005). "Discrimination of Primary and Secondary Dengue Virus Infection by an Immunoglobulin G Avidity Test Using a Single Acute Phase Serum Sample," Journal of Clinical Microbiology, 43, 2793-2797.

Murgue, B., Deparis, X., Chungue, E., Cassar, 0. \& Roche, C. (1999). "Dengue: An Evaluation of Dengue Severity in French Polynesia Based on an Analysis of 403 Laboratory-Confirmed Cases," Tropical Medicine and International Health, 4, 765-773.

Norman, G., Theodre, A. \& Joseph, A. (1991). "An Insular Outbreak of Dengue Fever in a Rural South Indian Village," Journal of Communicable Diseases, 23, 185-190.

Pavri, K. M. (1978). 'Ecology of MosquitoBorne Viruses in India and Southeast Asia in: Loutit MW, Miles JAR,' Eds. Microbial Ecology. Berlin.

Pérez-Guerra, C. L., Seda, H., García-Rivera, E. J. \& Clark, G. G. (2005). "Knowledge and Attitudes in Puerto Rico Concerning Dengue Prevention," American Journal of Public Health, 17(4), 243-252.

Silva, E. F., Orsi, M., Andrade, A. L., Domingues, R. Z., Silva, B. M., de Araújo, H. R. C., Pimenta, P. F. P., Diamond, M. S., Rocha, E. S. O., Kroon, E. G., Malaquias, L. C. C. \& Coelho, L. F. L. (2012). "A Tetravalent Dengue Nanoparticle Stimulates Antibody Production 
11 Journal of Virology \& Microbiology

in Mice," Journal of Nanobiotechnology, 10,

13.

Strobel, M. \& Lamaury, I. (2001). "Fièvre Dengue: Mise au Point," Revue De Médecine Interne, 22, 638-647.

Teixeira, M. G., Barreto, M. L., Costa, M. C. N., Denize, L., Ferreira, A., Vasconcelos, P. F. C. \& Cairncross, S. (2002). "Dynamics of Dengue Virus Circulation: A Silent Epidemic in a Complex Urban Area," Tropical Medicine and International Health, 7, 757-762.

Tripathi, P., Kumar, R., Tripathi, S., Tambe, J. J. \& Venkatesh, V. (2008). "Descriptive Epidemiology of Dengue Transmission in Uttar Pradesh," Indian Pediatrics, 45, 315318. 